\title{
“Oops, I didn't mean to be so flippant" \\ A corpus pragmatic analysis of apologies in blog data
}

\begin{abstract}
This chapter studies the form oops and its function as an Illocutionary Force Indicating Device (IFID) signalling apologies in a corpus of blog posts and reader comments. The focus is on the adaptability of speech acts to online media and the implications for the formal choice of linguistic expressions beyond the prototypical examples of routinised apology IFIDs. Thus, this study takes a closer look at the pragmatic functions of oops in the Birmingham Blog Corpus, a diachronically-structured collection covering the period 2000-2010, to gain new insights into its use and distribution.
\end{abstract}

Keywords: apology, speech act, blogs, CMC, collocation, corpus linguistics

\section{Introduction}

This paper examines the adaptability of speech acts (Searle, 1979) to new media by adopting a corpus linguistic approach. In particular, it addresses the use and distribution of the speech act of apologising in a large corpus of blog posts and reader comments, illustrating how this speech act has undergone development with regard to the formal choice of linguistic expressions in this specific environment which allows for interactivity and communicative immediacy.

This work builds upon previous research where we tried to uncover the ways in which users apologise in blogs, and derives from one of the first large-scale analyses of the Birmingham Blog Corpus (BBC): a diachronically-structured collection covering the period 2000-2010 and totalling 600 million words. We took an initial list of Illocutionary Force Indicating Devices (IFIDs) associated with the speech act of apology, and used a novel form of collocational analysis to reveal "hidden manifestations" (Kohnen, 2007) of apologies in the $B B C$. We were able to achieve this without relying on external thesauri by examining the shared and unique collocates of each of our initial apology IFIDs. As a consequence, we discovered that the form oops, together with its many orthographic variants, is frequently attested with an apology function in our data, illustrating the structural and contextual adaptability of the speech act of apologising.

In this paper, we look more closely at the uses of oops as an apology IFID in the $B B C$. Our initial analyses suggest that, although oops is often accompanied by a prototypical apology 
IFID such as sorry, this is not always the case and it appears that oops may be acting as an apology IFID in its own right in blog data. Thus, this paper will offer new insights into the nature of oops and its pragmatic functions, and make an important contribution to the wider debate on the role of speech acts in online data.

\section{Defining and identifying apologies}

Apologies are a type of speech act which plays an important role in human life. They are a means of expressing politeness and their use is significant on a social, cultural and psychological level. At its core, an apology implies that some wrongdoing or offence has occurred which, in accordance with social and cultural norms, requires remedial action. By uttering an apology, the speaker acknowledges this breach of norms and, according to the definitional criteria of an apology, must take responsibility for the offence and express regret (see e.g. Fraser, 1981, Wierzbicka, 1987); it is then up to the hearer to interpret the speech act accordingly, or not.

An apology can thus be defined in general terms as a "[j]ustification, explanation, or excuse, of an incident or course of action" (OED: s.v. apology). More specifically, an apology “is a speech act addressed to B's face-needs and intended to remedy an offense for which A takes responsibility, and thus to restore equilibrium between $\mathrm{A}$ and $\mathrm{B}$ (where $\mathrm{A}$ is the apologizer, and B is the person offended)" (Holmes, 1990: 159). A prototypical apology therefore includes four components according to Deutschmann (2003: 44ff.): an offender who recognises and takes responsibility for an offence whose victim was the offended and the ensuing remedy leading to an expression of regret.

Due to their significant nature as "one of the most ubiquitous and frequent 'speech acts' in public discourse and in social interactions" (Drew et al., 2016: 1), apologies have received continued attention in academic research. Thus they have recently been the focus of a special issue of the journal Discourse Processes, where all contributors studied "the same corpus of apologies in (US and UK) English telephone calls" (Drew et al., 2016: 3), resulting in analyses of parasitic apologies (Galatolo, Ursi and Bongelli, 2016), responsibility and culpability in apologies (Fatigante et al., 2016), or the construction "I'm sorry about that" (Margutti, Traverso and Pugliese, 2016). In addition to telephone conversations, studies have explored the use of apologies in emails (Harrison and Allton, 2013), on Twitter (Page, 2014), in different languages and politeness cultures (Tanaka, Spencer-Oatey and Cray, 2008, Ogiermann 2009), and in public and political contexts (Kampf and Löwenheim, 2012, Benoit, 2014). 
In speech act theory, apologies are attributed to the speech act category of expressives which convey the speaker's feelings, as for example, by thanking, praising, or apologising (Searle, 1976: 12-13, 1979: 15-16). In this paper, we focus on one type of expressive which is realised in highly routinised ways by a conventionalized set of forms in Present Day English (see also Deutschmann, 2003: 36). Holmes (1990: 175) describes apologies as "remarkably formulaic speech acts: a very small number of lexical items and syntactic patterns account for the great majority of the explicit apologies". These explicit apologies are characterised by their use of specific IFIDs, that is forms which usually indicate a particular illocutionary force, in this case the communicative force of apology.

Aijmer (1996: 94-95) regards the occurrence of the apology strategy 'explicit apologizing' as a necessary or core component in the apology strategy patterns that she identified in the London-Lund Corpus. This is further supported by Meier (1998: 216), who claims that " $[\mathrm{t}]$ he most frequently occurring apology strategy has generally been found to be a formulaic expression of apology (i.e. an expression containing apologize, sorry, forgive, excuse, pardon)", with sorry being "the overwhelming favorite" (see also Blum-Kulka and Olshtain, 1984: 206-207, Wierzbicka, 1987: 217). Thus, it has been found that in English speakers tend to apologise by using an IFID from a set of routine expressions and only rarely apologise without using any of these forms. Consequently, identifying apologies in corpora is, as Deutschmann (2003: 36) notes, "relatively easy".

Deutschmann (2003) carried out a corpus-based study of apologies in that he analysed a list of IFIDs in a sub-section of the spoken component of the British National Corpus (BNC) comprising about five million words of dialogue between speakers of known age and gender. He focused in particular on "expressions containing variants of the words afraid, apologise, apology, excuse, forgive, pardon, regret and sorry" (2003: 18), basing this selection on the Cross Cultural Speech Act Realization Pattern (CCSARP) taxonomy (cf. e.g. Blum-Kulka and Olshtain, 1984) and manually examining matches to identify explicit apologies. The same approach was adopted by Page (2014) in her study of corporate apologies on Twitter, but she coded only a small subset for types of apology.

In the case of the speech act of apologies, previous studies have thus often used IFIDs or speech act verbs as their starting point. This was done in the understanding that the output obtained would not necessarily represent a complete list of all attestations of the speech act in a data set but it was "likely to be a fairly substantial subset, [...] a very important subset, [...] because the IFID constitutes the routinized and lexicalized expression of an apology" (Jucker and Taavitsainen, 2008: 233). At the same time, this approach is suitable for corpus linguistic 
investigations using search tools that require the input of specific linguistic forms or phrases. While thus facilitating automated corpus linguistic searches of large corpora, any attestations of apologies not using one of the IFIDs in question or not lexically signalled will, however, be missed in a study based on a finite set of search terms. In other words, one could say that it is unlikely to be possible to extract all attestations of a speech act using a quantitative corpus linguistic methodology, as opposed to qualitative analyses based on close reading of texts or ethnographic approaches to the study of speech acts, where a wider range of realisations is likely to be identified.

Nevertheless, we would argue that the gap between the different methodological approaches can be bridged, to some extent at least, and corpus linguistic tools can be used as a means of detecting additional ways of apologising that have not been included in the list of routine expressions mentioned above. In the current study, we show how the study of a large corpus, for which a close reading analysis is not feasible, can go beyond the use of a finite list of IFIDs in the study of apologies. In contrast to previous approaches that focused on standard routine expressions in corpora of online data (see e.g. Harrison and Allton, 2013, Page, 2014), our work demonstrates that referring to shared and unique collocates can uncover further uses of apologies, as we will discuss below.

At the same time, we argue that these additional forms may be an indication that the speech act is adapting to the environment in which it occurs, that is online data and blogs in particular. We show how, in an online environment, additional forms that were not discussed in previous studies are used frequently with an apology function, which could be interpreted as the speech act's adaptability to the medium. According to Verschueren's notion of adaptability (1999: 66-68), which he regards as a key notion in pragmatic study and explanation, that is to say that our study takes the contextual correlates and structural objects of adaptability into account by investigating the interplay between a specific pragmatic phenomenon (the speech act of apologising) and its context of occurrence (the medium of the blog). We start by discussing our data in Section 3, highlighting the main features of our corpus and the medium of the blog. In Sections 4 and 5, we then explain how the collocational analysis of apology IFIDs has revealed the form oops and engage in an analysis of oops in our blog data, demonstrating how it can have an apology function when co-occurring with other apology IFIDs but also when appearing on its own. As a consequence, this paper also touches on Verschueren's "dynamics of adaptability" (1999: 69), which he defines as "the unfolding of adaptive processes in interaction", as it explores a specific linguistic form or structure and its evolving relationship with its context or medium. 


\section{Data}

Our study is based on the Birmingham Blog Corpus $(B B C)$, a diachronically-structured collection covering the period 2000-2010 and totalling 630 million words. The corpus is freely available and searchable through the WebCorp Linguist's Search Engine (WebCorpLSE) software built by the Research and Development Unit for English Studies (RDUES) at http://www.webcorp.org.uk/blogs. In this paper, we focus on a 181 million word sub-corpus taken from the WordPress and Blogger hosting sites. ${ }^{1}$ Such sites offer the advantage that, although the various blogs hosted there may look very different and cover a diverse range of topics, there is actually a limited set of templates available to users, which makes it possible to write software tools to extract textual content by analysing the HTML code of each blog (see Kehoe and Gee, 2012). Importantly for our purposes, the regular nature of WordPress and Blogger allows for the separation of blog posts from reader comments on those posts, and of individual comments from one another. It is also possible to determine exactly when each post and comment was written, which is unusual for web data.

The WordPress and Blogger sub-corpus of the $B B C$ was built by taking as a starting point the 'trending' blogs on each hosting site, known as 'Blogs of Note' on Blogger and 'Freshly Pressed' on WordPress. As described in more detail in Kehoe and Gee (2012), we used our WebCorpLSE tools to download each post from each of the featured blogs, and to extract the textual content from each post together with the reader comments associated with it. During the process, we found hyperlinks to other WordPress and Blogger blogs and added these to the queue for crawling, thus widening our coverage beyond the initial 'trending' list. During a one month crawl we downloaded 222,245 blog posts from WordPress and Blogger combined, totalling 95 million tokens. In addition, we downloaded all reader comments on these posts, making a further 86 million tokens. This gives us a corpus of 181 million tokens overall, and it is significant that there are almost as many tokens in the comments on the posts as there are in the posts themselves. We indicate in our analysis in Section 5 whether particular examples occur in a post or in a comment where this is relevant to the point being made.

Preliminary analyses of the $B B C$ (Kehoe and Gee, 2012) have already provided evidence of the increasingly diverse nature of blog posts. This research revealed that the established definition of blogs as online journals or diaries - found in many dictionaries - is now outdated and there are many uses to which the blog format is put. While there remain many blogs where a single author reflects on his or her own life and interests, there also exist large corporate blogs with multiple contributors and vast audiences. Kehoe and Gee (2012) noted a trend for 
dedicated blog sections on news websites, paying particular attention to the UK newspaper The Guardian. They also found, through an analysis of Google hit counts, that there are more blog posts hosted on Blogger than there are web pages from any single country outside the United States.

Blogs in general are an interactive medium as they allow the author of a blog post to communicate his or her message and readers of the post to comment on it. While facilitating such interactivity, the nature of blogs does not, however, inherently request it and, contrary to other media, a reply or comment is not necessarily provided. At the same time, the medium allows for a theoretically infinite time lag between post and comment, therefore creating “interaction-at-one-remove" (Nardi et al., 2004: 46). As boyd (2006) points out "[t]he practice of blogging involves producing digital content with the intention of sharing it asynchronously with a conceptualized audience". It is an n-to-? practice, which means that a known number of bloggers, usually one but it may also be a group, communicates with an unknown number of readers. Based on the intended readership, i.e. the specific conceptualization of the audience, blogs may show features traditionally associated with spoken language and communicative immediacy (see Koch, 1999). Consequently, they may also contain language innovations which may already appear in speech but may not yet be attested in more conventional written texts (see e.g. Renouf and Kehoe, 2013 on the new adjectival use of genius, as in a genius idea, being significantly more frequent in the BBC than in other written corpora).

\section{Collocational analysis of apology IFIDs}

When beginning our research on apologies, our aim was to start with an initial list of apology IFIDs taken from Deutschmann (2003), and then to expand this list through collocational analysis. Our approach was based on the notion that the meaning of a word can be determined by its immediate textual environment or that "[y]ou shall know a word by the company it keeps" (Firth, 1956: 11). We started with a list of words we assumed to be semantically-related - the apology IFIDs - and used collocational analysis to examine overlaps and differences in their meaning in more detail. The initial list contained the IFIDs sorry, pardon, excuse, afraid, apologi(s/z)e, forgive, regret, and apology, including associated inflections. Our hope was that further apology IFIDs would emerge in the process of collocational analysis and, thus, that through this approach we would uncover previously "hidden manifestations" (Kohnen, 2007: 141) of apologies.

We were particularly interested in the unique collocates of each apology IFID: words which appear frequently in close proximity to that IFID in our corpus but which do not appear 
in close proximity to any other apology IFID. We defined close proximity as a window of four words to the left and four words to the right of the IFID. We took the lists of the top 100 collocates for each of the eight apology IFIDs, sorted by collocational strength (z-score), and compared these lists with one another to find unique collocates. It was through our analysis of the unique collocates of the IFID sorry that we uncovered the word oops. The top 20 unique collocates of sorry, sorted by strength of collocation with sorry, were as follows:

$\begin{array}{llll}\text { hear } & \text { feeling } & \text { sick } & \text { ooops } \\ \text { about } & \text { bout } & \text { sucks } & \text { hugs } \\ \text { delayed } & \text { rambling } & \text { couldn't } & \text { aww } \\ \text { oops } & \text { disappoint } & \text { lost } & \text { hon } \\ \text { haven't } & \text { forgot } & \text { happened } & \text { hun }\end{array}$

That is to say that none of these words appears in the top 100 collocates of any of the other seven apology IFIDs. As will be noted, there are actually two orthographic variants of oops within the top 20 unique collocates of sorry: oops and ooops. We go on to investigate this further in the following section.

\section{Oops!}

After noting the appearance of the two variants of oops in the unique collocates list for sorry, we wanted to analyse this word in more depth to assess its potential as an apology IFID. Some previous studies of apologies have made brief mention of oops. For example, Holmes (1990: 16) includes it in a "potentially infinite" list of words and phrases which "might serve as an apology in an appropriate context and with appropriate intonation and paralinguistic support". She does not, however, analyse oops in more detail. Likewise, the OED (s.v. oops, int. and n.) mentions oops as "expressing apology" especially "after an obvious but usually minor mistake", with the first attestations of this use going back to the beginning of the twentieth century. On the other hand, Ogiermann (2009: 124f.) mentions oops in her discussion of exclamations that may precede an apology IFID and primarily express surprise or emphasise the nonintentionality of an event, rather than functioning as an apology in their own right. Stange (2016) in her study of emotive interjections also discusses whoops as an interjection of surprise. While acknowledging that it can function as a spill cry (cf. Goffman, 1978) and be used for minor mishaps, she discusses whoops with reference to its surprise function only. 
To carry out a full analysis of oops in blogs, we searched the BBC sub-corpus for other variants using the search term $o o^{*} p s$ in WebCorpLSE (that is, 'o' followed by one or more 'o's, followed by 'ps' - case insensitive). In addition to oops and ooops, we found 42 other orthographic variants, with a total corpus frequency of 3,891 . The most frequent variants are:

Oops (1816), oops (1321), Ooops (309), ooops (145), OOPS (106), Oooops (44), Oops (34), oooops (25), Ooooops (11), OOOPS (8)

The capital letter at the beginning of the most frequent and several of the other variants reflects the regular appearance of oops at the beginning of a sentence (see Figure 1 for examples). In fact, in $72 \%$ of cases in our corpus, $o o^{*} p s$ appears as the first (or only) word in a sentence.

In addition to $o o^{*} p s$, we found further orthographic variants beginning with 'w' or 'wh', with a total corpus frequency of 798. This gave us 4,689 instances of all variants of $(w(h)) o o^{*} p s$ combined. It is worth pointing out here that, although the count for the whoops variant includes irrelevant examples such as whoops of joy, that exact phrase appears only once in our corpus and there are only four other examples of whoops of X (including cop cars, breath, and delight). In what follows, we use collocational analysis to focus on the use of whoops as a variant of oops, which accounts for the vast majority of its 667 occurrences. Using WebCorpLSE, we took all orthographic variants of oops together and extracted their top collocates at span 4 (see Table 1). This collocation table includes additional columns showing the frequency of each collocate in each position in the span 4 window, where L1 is the position immediately before oops, $\mathrm{R} 1$ is the position immediately after, and so on. The total collocational frequency is given in the centre, with the z-score - a measure of collocational significance - on the right. In what follows, we use the term oops to refer to all orthographic variants combined. 
Table 1: Collocates of oops - all orthographic variants (span 4)

\begin{tabular}{|l|r|r|r|r|r|r|r|r|r|r|}
\hline Word & $\mathbf{L} 4$ & $\mathbf{L 3}$ & $\mathbf{L} 2$ & $\mathbf{L 1}$ & Total & $\mathbf{R} 1$ & $\mathbf{R 2}$ & $\mathbf{R 3}$ & $\mathbf{R 4}$ & Z-score \\
\hline forgot & 7 & 5 & 0 & 4 & 308 & 145 & 116 & 25 & 6 & 193.07 \\
\hline meant & 5 & & 2 & 1 & 206 & 45 & 120 & 23 & 10 & 98.46 \\
\hline sorry & 3 & 2 & 1 & 1 & 308 & 260 & 21 & 13 & 7 & 82.74 \\
\hline mean & 1 & 1 & 4 & 4 & 182 & 0 & 139 & 22 & 11 & 46.83 \\
\hline I & 105 & 71 & 33 & 8 & 1968 & 1011 & 334 & 223 & 183 & 26.82 \\
\hline typo & 1 & 0 & 0 & 2 & 27 & 19 & 1 & 1 & 3 & 24.87 \\
\hline wrong & 6 & 2 & 9 & 2 & 77 & 25 & 3 & 14 & 16 & 23.61 \\
\hline say & 10 & 4 & 1 & 12 & 166 & 0 & 4 & 78 & 57 & 20.69 \\
\hline missed & 2 & 0 & 4 & 0 & 51 & 11 & 24 & 4 & 6 & 20.41 \\
\hline supposed & 1 & 2 & 1 & 0 & 39 & 1 & 6 & 22 & 6 & 18.94 \\
\hline comment & 6 & 6 & 4 & 8 & 72 & 3 & 8 & 15 & 22 & 15.91 \\
\hline didn't & 5 & 5 & 1 & 0 & 111 & 21 & 52 & 19 & 8 & 15.88 \\
\hline did & 7 & 1 & 5 & 4 & 144 & 66 & 36 & 18 & 7 & 15.41 \\
\hline mention & 3 & 1 & 3 & 0 & 36 & 0 & 1 & 17 & 11 & 15.15 \\
\hline mistake & 0 & 0 & 0 & 4 & 20 & 1 & 9 & 2 & 4 & 13.58 \\
\hline should & 5 & 0 & 1 & 1 & 99 & 20 & 46 & 13 & 8 & 12.75 \\
\hline email & 0 & 1 & 2 & 1 & 38 & 4 & 7 & 16 & 7 & 12.59 \\
\hline posted & 3 & 3 & 4 & 2 & 37 & 5 & 9 & 7 & 4 & 12.46 \\
\hline accidentally & 0 & 2 & 0 & 0 & 15 & 3 & 7 & 3 & 0 & 12.33 \\
\hline lol & 2 & 1 & 12 & 18 & 58 & 14 & 3 & 3 & 5 & 12.29 \\
\hline bad & 4 & 6 & 4 & 1 & 58 & 12 & 21 & 3 & 7 & 11.70 \\
\hline
\end{tabular}

We see here that forgot is the most significant collocate of oops overall, appearing most frequently in R1 and R2 positions. Upon checking with WebCorpLSE we find that, where forgot appears in R2 position, the intermediate word (i.e. the word in the R1 slot) is usually the first person pronoun (in $82 \%$ of cases). We explore this further in Figure 2 below. Indeed, in raw frequency terms, $I$ is the most common collocate of oops overall, especially in the R1 slot (1011 instances). Where forgot appears in the R1 slot, this is usually because the first person pronoun has been ellipted (e.g. "Oops, forgot the time difference").

Table 1 also offers an initial indication of the kinds of thing for which people apologise in our blog corpus, namely errors (e.g. wrong, mistake, typo) and omissions (e.g. forgot, mean $(t)$, missed). It is difficult to draw any conclusions about these words out of context so we go on to explore some examples in more detail in the remainder of this section, before returning to bad in Section 6.

One thing that is particularly striking about Table 1 is that, although we have previously shown oops to be a strong collocate of sorry, in only 308 (7\%) of the 4,689 occurrences of oops does sorry appear within four words to the left or right. Most of its attestations appear in the 
first position to the right of oops. This is illustrated in Figure 1, which lists examples of oops followed immediately by sorry.

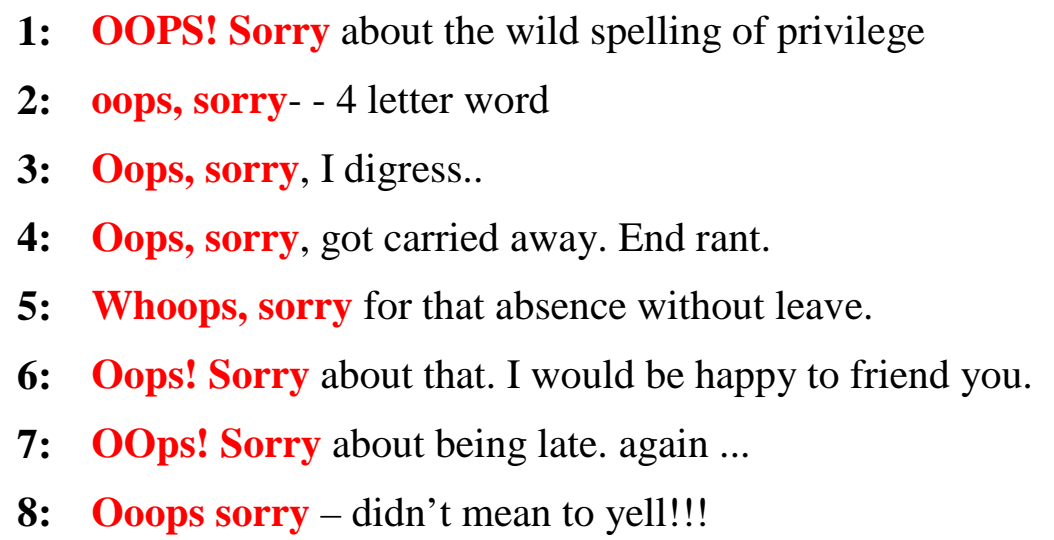

Figure 1: Examples of oops (all variants) collocating with sorry in R1

As Figure 1 shows, oops either occurs in sentence initial position or makes up its own syntactic unit, being followed by a full stop or an exclamation mark. Its apologetic illocutionary force is here reinforced by the prototypical IFID sorry and the examples illustrate some of the specific reasons for which bloggers and/or commenters apologise. Examples 5 and 7 contain apologies for absence or lateness in posting, example 1 apologises for misspelling the word privilege in a previous comment, and examples 3 and 4 are apologies for writing too much. Thus, one can see that some of the apologies voiced are related to the medium of the blog, which is asynchronous in nature but may have certain implied expectations regarding the frequency of posts and where replies can be linked to specific comments. At the same time, examples 2 and 8 show that the medium can also be exploited to create a certain effect such as imitating speech by yelling (example 8) or sarcasm through posting a comment that could have been deleted before publication (example 2).

However, as we indicated above, the form oops does not always collocate with sorry or another routinised apology IFID when serving the function of an apology. This is illustrated in Figure 2, where the first three examples show oops collocating with sorry and the past tense form of the verb forget, which according to Table 1 has the strongest collocational link with oops in our data. Examples 4 to 6, on the other hand, show that oops is also attested in collocation with I forgot when sorry is not found within a collocational span of four words to the left or right.

1: Oops, sorry, I forgot, you can't see me 


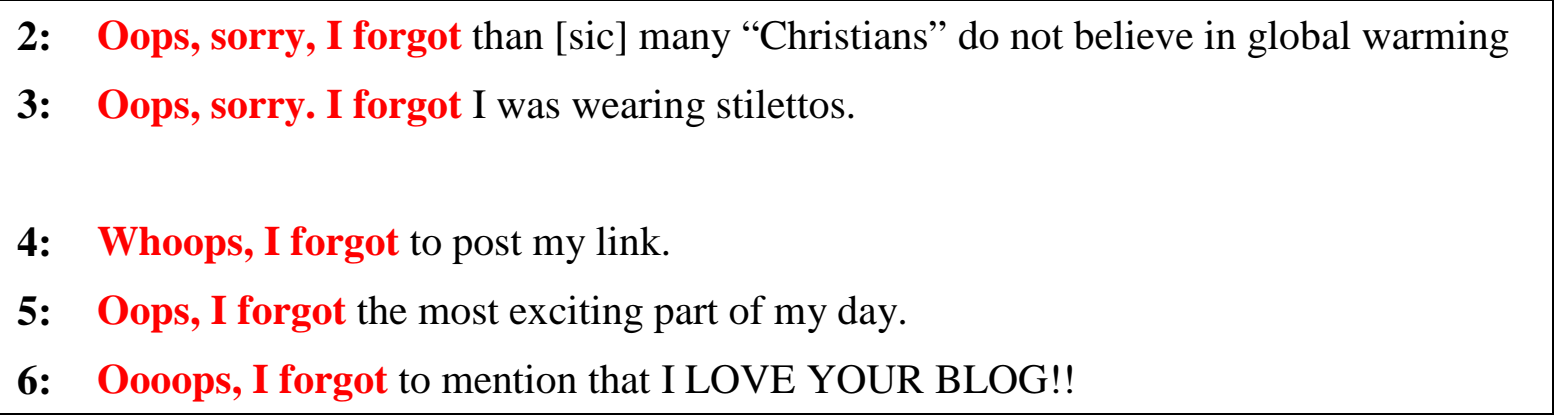
Figure 2: Examples of oops (all variants) in sentence initial position with and without sorry within a collocational span of 4 words to the left or right

In some of the examples in Figure 2 oops is used to apologise for a genuine oversight. For instance, example 4 appears in a comment on a post about a prize draw being offered by a blogger to celebrate reaching 400 followers. ${ }^{2}$ The commenter, called Laurel, had originally commented thirteen minutes earlier promising to share details of the draw on her own blog. She then posted the follow up comment given as example 4 to share the link to her own blog post. Likewise, in example 6, the user left a positive comment, expressing appreciation for a post and its content, only to add a further comment two minutes later extending this appreciation to the whole blog.

In example 5, oops appears in a blog post ${ }^{3}$ in which the blogger has already described her birthday and now, when closing her post, remembers that she has not yet mentioned the most exciting part of her day. This example illustrates the conversational nature of the blog and its similarity to spoken accounts as the user could have edited her post to emphasise the importance of this part of her day but decided not to, attaching its narration to the end of the post as (if) it had slipped her mind earlier. In all of these examples, oops has some apologetic illocutionary force, with users apologising for not having mentioned something earlier or for elaborating on a specific point instead of closing a post.

On the other hand, our corpus also includes examples such as 1-3 in Figure 2, where oops collocates with the prototypical apology IFID sorry but "where the remedial nature of the apology is questionable" (Deutschmann, 2003: 46). This is because the context in which the IFID is used is, for instance, sarcastic or challenging the addressee, which in the case of blogs may be individuals or the whole blogging community. Deutschmann (2003) refers to this type of apology as 'face attack apologies' as their level of sincerity does not meet the conditions of a prototypical apology, where regret for an offence has to be shown. This is illustrated in examples 1-3 in Figure 2. Example 2 comes from a comment thread on a post entitled "What is the Bible?" by a professor of religious studies at Rice University, in which she quotes one of 
her student's answers to this question. ${ }^{4}$ In this example, a commenter elaborates on the student's view that the bible is interpreted in ways that support certain value and belief systems by linking the reinterpretation of Christian values to the Republican Party and stressing that the Republican values are "completely anti-Christian". The forms oops and sorry then introduce a sarcastic remark rather than an expression of regret, underlined by the quotation marks surrounding Christians, and therefore serve a face attack function, directed at the wider Christian community that disrespects core Christian values. Example 1 is similarly sarcastic, as readers of a blog post clearly cannot see the poster, and example 3 comes from a post in which a blogger engages in a fictional interaction with a personification of 'the Holidays' where she attempts to throw them out of the back door but fails as she stumbles because she is wearing stilettos, underlining the constructed and therefore insincere nature of oops sorry. ${ }^{5}$

As the examples in Figure 2 have illustrated, our corpus shows that oops is attested as an apology IFID when collocating with sorry and when appearing on its own. As for its specific apology functions, one may note that oops tends to diverge from the prototypical apology category by functioning as a face attack apology (as discussed above but see also example 5 in Figure 3 ) or by being of a more formulaic nature. This is illustrated by examples of bloggers apologising for typos or for using the wrong word, which could potentially be described as the written equivalents of slips of the tongue. At the same time, oops also seems to be serving additional discourse marking functions when acting as a formulaic apology (cf. Deutschmann's (2003: 46) category of 'formulaic apologies with added functions'). This is because oops tends to initiate a new section of a blog post or a comment that may require some degree of hedging in order to reduce the abruptness of the transition. This is shown in Figure 3, where oops introduces examples of rather minimal offences and functions as a routine apology in addition to beginning a new section of text. Thus, example 1 introduces the main body of a post after an introduction that turned out longer than intended, and oops in example 3 initiates a metapragmatic comment on the poster's own language use.

1: Whoops, that intro got longer than I intended.
2: Oops, I didn't mean to be so flippant.
3: Ooops! I just sounded like my teenager. lol!
4: Oops I forgot to say CONGRATULATIONS!
5: Ooooops! I just told the punch line before the joke.
6: Whoops. I meant to say "stare," not "state" in my previous comment.
7: Oops ... I meant to say 'What an inspirational post, Laura!' 
8: Oops! I didn't mean to post the comment twice!

Figure 3: Examples of oops (all variants) in sentence initial position where sorry does not appear within a collocational span of 4 words to the left or right

As we noted previously, $72 \%$ of all instances of oops in our corpus appear as the first (or only) word in a sentence. It follows, then, that oops is often the first word in a comment. In fact, we find that oops tends to appear at the beginning of a user's second comment, as a correction or addition to a previous comment, often one that was made only a few minutes ago. This is illustrated in examples 2, 4, 6, 7 and 8 in Figure 3, which are all taken from the beginning of a user's comment on one of their previous comments on the same post. Thus, in example 2 the commenter clicked on publish too quickly and therefore apologises for being "so flippant", in example 6 they correct one of their misspellings, in example 7 the user had initially referred to Laura as Jennifer accidentally, and in example 8 the user posted the same comment twice by mistake. Example 4 is quoted with its surrounding context below:

Post:

I started this blog on Aug 12010 today I reached a hundred followers on my blog, you are all amazing. And we shall have a giveaway as soon as I recover from this idiotic flu. The son just passed it to me and I am sure I will be passing it right back bcos its just us two at home.

Comments:

Madeleine said... Poor you, hope you feel better soon :O) October 28, 2010 9:08 AM

Madeleine said... Oops I forgot to say CONGRATULATIONS! October 28, 2010 9:08 AM

As this example shows, the original post ${ }^{6}$ mentioned two coinciding events: the user reached a hundred followers and it happened on a day that she had the flu. The commenter Madeleine referred only to her illness in her first comment, wishing the user a quick recovery. Immediately afterwards, as indicated by the time stamp, she posted a second comment to express her congratulations on the one hundred followers. This and similar examples suggest that oops in its formulaic apology function has a medium-specific use in blogs, where it introduces a user's second comment, intended to rectify a mistake or add information that has been previously forgotten. This type of apology was also identified by Harrison and Allton (2013: 327) in their corpus of email apologies and referred to as "retrospective apologies for minor offences". While they mention oops in their discussion of these apologies, they assign the apologetic function to its collocate sorry only, with oops indicating the accidental nature of the mistake. In contrast to Harrison and Allton (2013) and to Goffman's initial treatment of oops as a response cry defining 
an event "as a mere accident" (Goffman, 1978: 801, see also 1981), we regard oops as having adopted and displaying the illocutionary force of an apology in our data. This is because in the written medium of the blog it has developed uses that go beyond that of an exclamatory interjection in speech and, instead of merely pointing at a mistake, it functions in a similar way to the IFID sorry and shows additional discourse marking functions, as our above examples have illustrated.

\section{My bad}

Two other collocates in Table 1 that we analysed in detail were mistake and bad, both of which appear most frequently in R2 position (i.e. two words to the right of oops) but with bad the more frequent of the two. In all but one of the 21 instances of bad as an R2 collocate of oops,

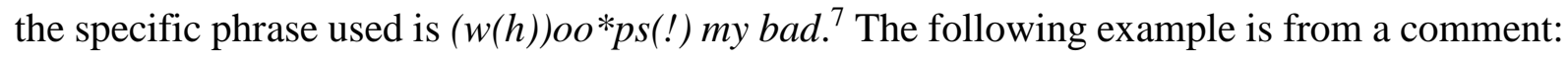

I must confess, I misread one of your sentences as "We'll discuss things like homophones..." as... "We'll discuss things like hormones..." Oops - my bad! (http://karenelange.blogspot.co.uk/2010/10/little-coffee-with-your-workshop.html)

The phrase my bad is used increasingly in colloquial English to mean 'my mistake', and is included in the OED (s.v. bad, adj., n.2, and adv.) with its earliest citations from 1985 and 1986:

4. colloq. (orig. U.S. Sport). With possessive: a person's fault; responsibility for a mistake, blunder, etc. Originally and chiefly in my bad (used mainly as int.).

1985 Gainesville (Florida) Sun 14 Nov. 3 e/2 Oops, my bad, I forgot for a moment what the Commodore did to Georgia. Silly me.

1986 C. Wielgus and A. Wolff Back-in-your-face Guide to Pick-up Basketball 226 My bad, an expression of contrition uttered after making a bad pass or missing an assignment.

Like oops, this phrase is potentially an apology IFID in its own right, signalling that the speaker/writer is admitting responsibility for some error. However, it is difficult to limit a corpus search to this particular use of bad. Grammatical restrictions cannot be applied to the search as the part-of-speech tagger underpinning WebCorpLSE (TreeTagger) does not allow bad to be tagged as a noun ${ }^{8}$, whereas a lexical search for my bad also includes spurious matches such as my bad luck and my bad back. However, the addition of oops helps to isolate the apology use of my bad. Indeed, our suggestion, based on the results from our collocational analysis, is that the IFID oops could be used as an additional filter to limit the instances of my bad to those where it is used as an apology. 


\section{Conclusion}

As Verschueren and Brisard (2002) have noted "language itself is the adaptable phenomenon in a context of changing technologies". This paper has provided evidence to support this observation through an analysis of the speech act of apologies in blogs. Using a 181 million word sub-corpus taken from the WordPress and Blogger hosting sites as the basis of our analysis, we have been able to show that the form oops is attested in various collocational contexts and with different functions in blog posts and comments.

Initially we had used collocation as a tool to reveal "hidden manifestations" (Kohnen, 2007) of speech acts. By carrying out a collocational analysis of the shared and unique collocates of a series of prototypical apology IFIDs, we uncovered the form oops appearing as a strong unique collocate of sorry. In this paper, we started by introducing the different spelling variations with which oops appears in our online data, illustrating the formal adaptability of this expression to the context in which it is used - variation that is not to the same extent referred to by the OED (s.v. oops, int. and n.) and may indeed point to a medium-specific use of the form. Taking a closer look at its functions showed that oops is used apologetically when collocating with sorry, which it does in only $7 \%$ of its attestations, but it also has this function when there is no apology IFID within its immediate collocational span. As a consequence, we would argue that oops functions as an apology IFID in its own right in our online data. It usually serves a formulaic apology function relating to minor offences such as typos and it shows additional discourse marking functions, appearing for instance repeatedly in comment-initial position. At the same time, we have been able to identify face attack apologies with oops, where the level of regret is low and the apology IFID introduces a sarcastic comment or a challenge.

Thus, while diverging to some extent from the prototypical apology, oops falls into these additional apology categories (see Deutschmann, 2003: 46) and on this basis we would argue that it offers an example of the formal adaptability of a speech act in the context of online data. That is to say that the form oops displays apologetic illocutionary force in the medium of the blog, in addition to the more routinized apology IFIDs but with the added stylistic effect of creating a conversational and colloquial impression in a written type of text, thus contributing to an increased perception of communicative immediacy. In fact, the different variants of oops appear with a frequency of 4,689 in the Birmingham Blog Corpus, which is more frequent than, for instance, the forms apology or pardon. Consequently, we would argue that the use of oops in blogs, and our brief discussion of my bad, provide some indication of the further development and adaptability of the speech act of apologising in online data. Future research is needed to 
assess whether these uses are specific to the medium of blogs or if they apply to online data more broadly.

\section{References}

Aijmer, Karin, 1996. Conversational Routines in English: Convention and Creativity. Longman, London.

BBC $=$ Birmingham Blog Corpus. 2010. Compiled by the Research and Development Unit for English Studies at Birmingham City University. http://www.webcorp.org.uk/blogs (last accessed 1 July 2016)

Benoit, William, 2014. Accounts, Excuses, and Apologies: Image Repair Theory and Research. Second edition. SUNY Press, Albany.

Blum-Kulka, Shoshana, Olshtain, Elite, 1984. Requests and apologies: A cross-cultural study of speech act realization patterns (CCSARP). Applied Linguistics 5 (3), 196-213.

boyd, danah, 2006. A blogger's blog: Exploring the definition of a medium. Reconstruction 6 (4).

Deutschmann, Mats, 2003. Apologising in British English. Umeå University, Umeå.

Drew, Paul, Hepburn, Alexa, Margutti, Piera, Galatolo, Renata, 2016. Introduction to the Special Issue on Apologies in Discourse. Discourse Processes 53 (1-2), 1-4.

Fatigante, Marilena, Biassoni, Federica, Marazzini, Francesca, Diadori, Pierangela, 2016. Responsibility and culpability in apologies: distinctive uses of 'sorry' versus 'I'm sorry' in apologizing. Discourse Processes 53 (1-2), 26-46.

Firth, John R., 1956. Papers in Linguistics 1934-1951. Oxford University Press, London.

Fraser, Bruce, 1981. On apologizing. In: Coulmas, Florian (Ed.), Conversational Routine. Explorations in Standardized Communication Situations and Prepatterned Speech. Mouton Publishers, The Hague, pp. 259-271.

Galatolo, Renata, Ursi, Biagio, Bongelli, Ramona, 2016. Parasitic apologies. Discourse Processes 53 (1-2), 97-113.

Goffman, Erving, 1978. Response cries. Language 54 (4), 787-815.

Goffman, Erving, 1981. Forms of Talk. University of Pennsylvania Press, Philadelphia.

Harrison, Sandra, Allton, Diane, 2013. Apologies in email discussions. In: Herring, Susan C., Stein, Dieter, Virtanen, Tuija (Eds.), Pragmatics of Computer-Mediated Communication. Walter de Gruyter, Berlin, pp. 315-337.

Holmes, Janet, 1990. Apologies in New Zealand English. Language in Society 19 (2), 155-199.

Jucker, Andreas H., Taavitsainen, Irma, 2008. Apologies in the history of English. Routinized and lexicalized expressions of responsibility and regret. In: Jucker, Andreas H., Taavitsainen, Irma (Eds.), Speech Acts in the History of English. John Benjamins, Amsterdam, pp. 229-244.

Kampf, Zohar, Löwenheim, Nava, 2012. Rituals of apology in the global arena. Security Dialogue 43 (1), 43-60.

Kehoe, Andrew, Gee, Matt, 2012. Reader comments as an aboutness indicator in online texts: introducing the Birmingham Blog Corpus. In: Oksefjell Ebeling, Signe, Ebeling, Jarle, Hasselgård, Hilde (Eds.), Studies in Variation, Contacts and Change in English 12: 
Aspects of Corpus Linguistics: Compilation, Annotation, Analysis. Proceedings of ICAME 32,VARIENG e-journal. University of Helsinki, Helsinki. http://www.helsinki.fi/varieng/series/volumes/12/kehoe_gee (last accessed 1 July 2016)

Koch, Peter, 1999. Court records and cartoons. Reflections of spontaneous dialogue in early Romance texts. In: Jucker, Andreas H., Fritz, Gerd, Lebsanft, Franz (Eds.), Historical Dialogue Analysis. John Benjamins, Amsterdam, pp. 399-429.

Kohnen, Thomas, 2007. Text types and the methodology of diachronic speech act analysis. In: Fitzmaurice, Susan, Taavitsainen, Irma (Eds.), Methods in Historical Pragmatics. Mouton de Gruyter, Berlin, pp. 139-166.

Margutti, Piera, Traverso, Véronique, Pugliese, Rosa, 2016. I'm sorry 'about that': apologies, indexicals, and (unnamed) offences. Discourse Processes 53 (1-2), 63-82.

Meier, Ardith J., 1998. Apologies: what do we know?. International Journal of Applied Linguistics 8 (2), 215-231.

Nardi, Bonnie A., Schiano, Diane J., Gumbrecht, Michelle, Swartz, Luke, 2004. Why we blog. Communications of the ACM 47 (12), 41-46.

OED. Oxford English Dictionary. http://www.oed.com/ (last accessed 1 July 2016)

Ogiermann, Eva, 2009. On Apologising in Negative and Positive Politeness Cultures. John Benjamins, Amsterdam.

Page, Ruth, 2014. Saying 'sorry': Corporate apologies posted to Twitter. Journal of Pragmatics $62,30-45$.

Renouf, Antoinette, Kehoe, Andrew, 2013. Filling the gaps: Using the WebCorp Linguist's Search Engine to supplement existing text resources. International Journal of Corpus Linguistics 18 (2), 167-198.

Searle, John, 1976. A classification of illocutionary acts. Language in Society 5 (1), 1-23.

Searle, John, 1979. Expression and Meaning. Studies in the Theory of Speech Acts. Cambridge University Press, Cambridge.

Stange, Ulrike, 2016. Emotive Interjections in British English. A Corpus-Based Study on Variation in Acquisition, Function and Usage. John Benjamins, Amsterdam.

Tanaka, Noriko, Spencer-Oatey, Helen, Cray, Ellen, 2008. Apologies in Japanese and English. In: Spencer-Oatey, Helen (Ed.), Culturally Speaking. Culture, Communication and Politeness Theory. Continuum, London, pp. 73-94.

Verschueren, Jef, 1999. Understanding Pragmatics. Arnold, London.

Verschueren, Jef, Brisard, Frank, 2002. Adaptability. In: Verschueren, Jef, Oestman, Jan-Ola, Blommaert, Jan, Bulcaen, Chris (Eds.), Handbook of Pragmatics Online. John Benjamins, Amsterdam.

Wierzbicka, Anna, 1987. English Speech Act Verbs. A Semantic Dictionary. Academic Press, Sydney.

\footnotetext{
${ }^{1}$ WordPress: http://www.wordpress.com/; Blogger: http://www.blogspot.com/ (last accessed 1 July 2016)

2 http://thealliterativeallomorph.blogspot.co.uk/2010/11/giveaway-time.html (last accessed 1 July 2016)

${ }^{3} \mathrm{http}: / /$ sassydog23.blogspot.co.uk/2009/10/october-23-1951-my-birthday.html (last accessed 1 July 2016)

${ }^{4}$ http://forbiddengospels.blogspot.co.uk/2010/05/what-is-bible.html (last accessed 1 July 2016)

5 http://rawknrobyn.blogspot.co.uk/2010/11/dear-holidays.html (last accessed 1 July 2016)

${ }^{6}$ http://joannastjames.blogspot.co.uk/2010/10/100-followers.html (last accessed 1 July 2016)
} 
7 The only exception is one instance of "oops! that's bad".

${ }^{8}$ Except as a proper noun, where "Michael Jackson's Bad", "Breaking Bad", "Nick Cave and the Bad Seeds" are all examples from our blog corpus. 